Cinémas

Revue d'études cinématographiques

Journal of Film Studies

\title{
La quête du père dans le film Sonatine de Micheline Lanctôt
}

\section{Josette Déléas}

Volume 8, numéro 1-2, automne 1997

Cinéma et mélancolie

URI : https://id.erudit.org/iderudit/024749ar

DOI : https://doi.org/10.7202/024749ar

Aller au sommaire du numéro

Éditeur(s)

Cinémas

ISSN

1181-6945 (imprimé)

1705-6500 (numérique)

Découvrir la revue

Citer cet article

Déléas, J. (1997). La quête du père dans le film Sonatine de Micheline Lanctôt. Cinémas, 8(1-2), 187-199. https://doi.org/10.7202/024749ar

\section{Résumé de l'article}

Cet article traite de la quête du père dans le film Sonatine de Micheline Lanctôt (1983). En reprenant cette métaphore de l'imaginaire québécois devenue obsédante, en littérature comme au cinéma, la cinéaste souligne clairement que la quête individuelle de ses personnages est en fait la même que la quête collective du Québec des années quatre-vingt, pays épris d'égalité sociale et de justice. Mais, contrairement à la société qui les entoure, les adolescentes de Sonatine ne sont pas sclérosées par la peur et l'indifférence auxquelles elles opposent toute la force de leur vouloir. Le monde qu'elles recherchent est un monde où l'anima et l'animus coexisteraient, où les pères ne seraient plus des patriarches, mais ressembleraient aux « secondes mères » de la famille matrilinéaire, et où la femme pourrait redevenir « l'avenir de l'homme ». 


\title{
La quête du père dans le film Sonatine de Micheline Lanctôt
}

\author{
Josette Déléas
}

\section{RÉSUMÉ}

Cet article traite de la quête du père dans le film Sonatine de Micheline Lanctôt (1983). En reprenant cette métaphore de l'imaginaire québécois devenue obsédante, en littérature comme au cinéma, la cinéaste souligne clairement que la quête individuelle de ses personnages est en fait la même que la quête collective du Québec des années quatre-vingt, pays épris d'égalité sociale et de justice. Mais, contrairement à la société qui les entoure, les adolescentes de Sonatine ne sont pas sclérosées par la peur et l'indifférence auxquelles elles opposent toute la force de leur vouloir. Le monde qu'elles recherchent est un monde où l'anima et l'animus coexisteraient, où les pères ne seraient plus des patriarches, mais ressembleraient aux "secondes mères" de la famille matrilinéaire, et où la femme pourrait redevenir "l'avenir de l'homme».

\section{ABSTRACT}

This article examines the quest for the father in the film Sonatine by Micheline Lanctôt (1983). By reinterpreting what has become a pervasive metaphor in the Quebec imaginary, both in literature and in cinema, the filmrnaker stresses that her individual characters' quest is, in fact, the same as Quebec's collective quest in the 1980 s, as a nation committed to social equality and justice. But unlike the society around them, the adolescent girls in Sonatine are not paralyzed by the fear and indifference which they oppose with all their force of will. The world which they seek is one in which anima and animus coexist, where the fathers are 
no longer patriarchs but resemble the "second mothers" of the matrilineal family, and where women can once again become the "future of humanity."

Dans son film Sonatine (1983), Micheline Lanctôt présente deux adolescentes montréalaises, Chantal et Louisette, perdues dans un monde indifférent où elles sont condamnées à vivre avec le seul soutien de l'amitié qui les unit. Lorsque l'amour qu'elles recherchent désespérément leur échappera, elles choisiront la mort au terme d'un parcours tragique qui, triste ironie, aura duré neuf mois. L'histoire que raconte Micheline Lanctôt est donc une histoire sombre, mais dissimulée derrière un titre dont le signifié n'a rien, lui, de dramatique. Dès la fin du générique, les deux adolescentes expliquent que sonatine est une "petite sonate en général assez facile». Cette définition accroche d'autant plus l'attention qu'elle est prononcée en voix off. $\mathrm{Ce}$ qu'elle souligne d'emblée, par l'utilisation de l'expression "en général ", c'est que le récit qui va suivre est celui d'une exception. Il l'est, dans la mesure où la quête amoureuse qu'il raconte n'est pas une simple aventure. C'est une quête essentielle et difficile qui, de surcroît, se démarque de celle des jeunes de l'ère postmoderne. Chantal et Louisette, certes, sont en proie à un désarroi auquel la jeunesse des grands centres urbains du monde entier peut s'identifier. Mais leur désir d'amour romantique correspond essentiellement au besoin de se trouver un père, besoin devenu la métaphore obsédante de l'imaginaire québécois, en littérature comme au cinéma. Nombreux sont les critiques qui partagent l'opinion de Ian Lockerbie selon qui « [...] au cinéma québécois l'amour romantique joue un rôle tout à fait mineur. Ici le lieu par excellence des rapports passionnels, c'est la famille, et le thème prééminent, c'est la quête $d u$ père ou de la mère" (p. 12). Ainsi en recherchant désespérément le père absent, Chantal et Louisette ressemblent étrangement aux "orphelins" qui, selon l'essayiste Heinz Weinmann (cité dans Harcourt, p. 62), hantent le cinéma québécois. Des "orphelins" dont l'angoisse ancestrale remonte au milieu du XVIII siècle alors que le roi de France, père suprême, abandonne son peuple pionnier. Ces orphelins se retrouvent aussi en littérature où, comme le 


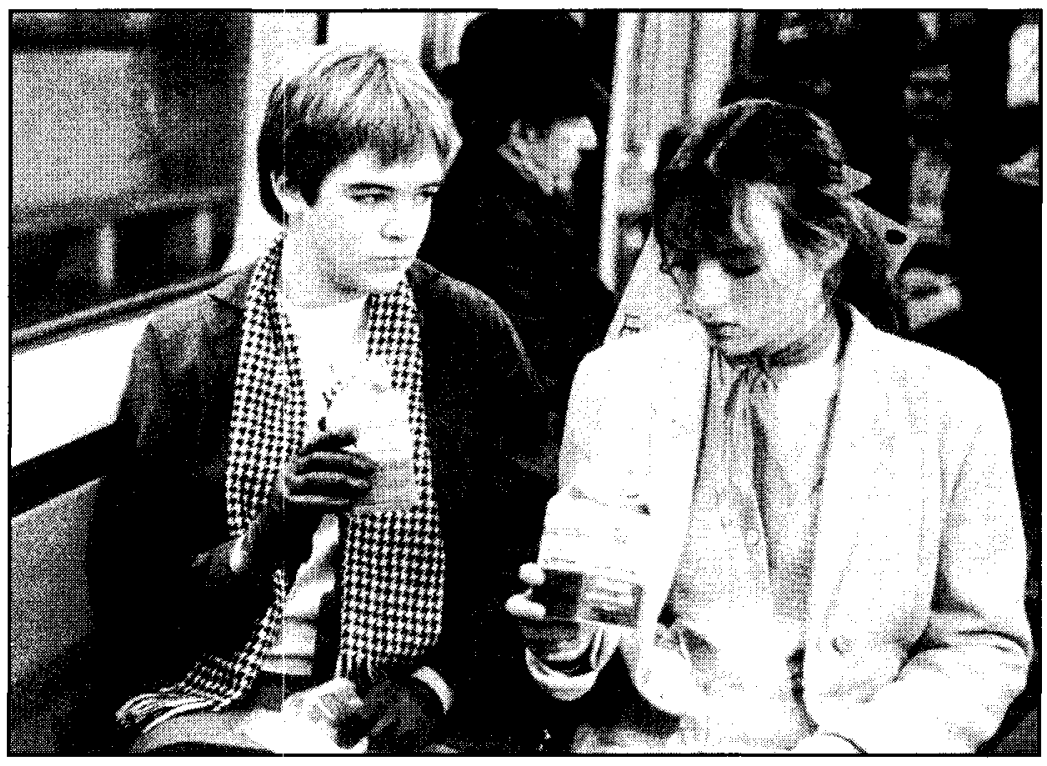

\section{Sonatine de Micheline Lanctôt (1983)}

Collection Cinémathèque québécoise

suggère prudemment le critique André Vanasse s'adressant à ses compatriotes, "[...] la fracture paternelle, c'est peut-être l'expression naturelle de notre fracture collective?" (p. 10).

Cette remarque permet d'établir un premier rapprochement de surface entre le titre du film et l'objet métaphorique du discours filmique. En effet, de même que la rupture avec la France a déterminé l'évolution historique du Québec et façonné l'inconscient collectif québécois, la forme sonate à laquelle s'apparente Sonatine a, elle aussi, joué un rôle historique influent mais, cette fois, bien évidemment, dans le domaine musical. Née en 1760, à l'heure du grand traumatisme originel d'un peuple abandonné par son Roi, la forme sonate, encore appelée «forme de premier mouvement" (De Candé, p. 108), allait servir de cadre à la plus grande partie de la musique instrumentale classique jusqu'au début du $\mathrm{XX}^{e}$ siècle.

Il est un autre rapprochement, plus important celui-ci, qui s'impose entre la structure du discours filmique de Sonatine et la structure de la forme sonate. Comme cette dernière, le récit de Micheline Lanctôt se compose de trois mouvements et de deux 
thèmes. Le premier "rapide, mobile'" retrace le parcours de Chantal, le second, "tendre, lancinant", celui de l'aventure de Louisette et enfin, le troisième, "disloqué, exaspéré", représente le vécu des adolescentes. Sur le plan historique, ces trois mouvements rappellent les trois grandes étapes de l'évolution du Québec ${ }^{2}$.

Le premier mouvement de Sonatine, "plein d'arrêts et de départs", est plongé dans une nuit où seul est éclairé l'intérieur de l'autobus, tantôt en mouvement, tantôt immobile. Chantal y poursuit, jusqu'au renoncement obligé, son rêve amoureux avec le chauffeur Fernand. Ce mouvement pourrait correspondre à la première étape de l'aventure collective québécoise, celle des temps pionniers où les rêves étaient grands mais ardus, les élans nombreux, mais finalement brisés par les défaites amères.

Le second mouvement, moins dynamique, plus «impressionniste", serait celui de l'époque où le Québec s'est cherché une identité. Ce mouvement est composé comme le précédent de séquences exclusivement nocturnes tournées sur un bateau amarré dans le port de Montréal. Il est tellement sombre qu'il est parfois impossible de distinguer les détails des plans. Il évoque ainsi, par analogie, l'entrée difficile du Québec dans la Confédération. Mais il rappelle surtout ce que Pierre Véronneau nomme "la morbidité collective" des années quarantecinquante, années de "la grande noirceur» durant lesquelles les Québécois étaient "[...] étouffés par les valeurs catholiques et ses ersatz: la souffrance, le châtiment, le péché, le sacrifice, la soumission» (p. 48).

Enfin, le troisième mouvement annonce la relève. Contrairement aux deux premiers, il est "ouverture" sur un monde de lumière. Les scènes de nuit sont ici remplacées par des scènes fortement éclairées. Mais ces scènes souffrent d'un autre type d'« enfermement". À l'exception d'une brève scène tournée dans la rue en plein jour (mais, précisons-le, sous un ciel pluvieux), ce dernier volet baigne dans l'éclairage artificiel du monde souterrain qu'est le métro de Montréal. Les deux adolescentes y passent le plus clair de leur temps lorsqu'elles ne sont pas chez l'une ou chez l'autre. Ce mouvement, explique Micheline Lanctôt, «[...] veut faire pénétrer le spectateur dans l'univers des deux 
adolescentes. Il est disloqué, exaspéré, excessif, il oscille entre le drame et la plaisanterie, il grouille d'émotions inarticulées, d'hyperboles, et nous mène par bonds imprévisibles jusqu'à la conclusion finale" (citée dans Blain, p. 144). Le rythme syncopé de ce dernier volet et le refus de séquences tournées en extérieur traduisent parfaitement les difficultés du changement et les risques d'échec auxquels se voit confronté le peuple québécois. Dans un monde privé des valeurs familiales rurales et de la tradition catholique, ce peuple doit découvrir les moyens de protéger les fondements de son identité s'il veut pouvoir l'afficher au grand jour sur le continent nord-américain. C'est à la jeunesse qu'incombe le rôle d'assurer cette sauvegarde en prenant la relève d'une collectivité sans cesse menacée dans son présent à cause d'un passé trop souvent occulté. En effet, s'il faut en croire Heinz Weinmann, le Québec est le pays qui "[...] "nationalise", institutionnalise pour ainsi dire l'oubli, l'oubli de son passé canadien-français» (cité dans Harcourt, p. 62). Ainsi, en accompagnant Chantal et Louisette, il sera permis d'identifier derrière leur quête individuelle, la quête collective du Québec des années quatre-vingt.

Entre les adolescentes de Sonatine et leurs parents, la rupture est totale. Les parents sont ici les grands absents. Ils n'apparaissent qu'une fois dans le film: ceux de Louisette pour se quereller autour d'elle sans la voir; ceux de Chantal pour la rejeter dans leur silence accusateur. L'unité familiale étant brisée, les jeunes filles orphelines se retrouvent sans passé. C'est pourquoi elles tentent de se constituer une mémoire, orientée vers un port d'attache, en enregistrant sur des cassettes, qui deviennent leur journal intime, les grands et les petits moments d'une existence qui ne va nulle part. Aussi, le baladeur, dont Chantal et Louisette ne se séparent jamais, occupe-t-il dans la diégèse un rôle de tout premier plan. Cette importance est annoncée dès le générique composé du seul gros plan du baladeur que, dans le hors-champ, Chantal et Louisette écoutent. Elles commentent en duo leurs propos décousus, teintés d'absurde, entrecoupés de rires et enveloppés dans les accents de la sonatine qui deviendra le leitmotiv musical du film. Cette longue séquence indique la tonalité du discours filmique qu'elle introduit. Elle signale 
d'entrée de jeu que les adolescentes se forgeront un passé, jour après jour, en l'inscrivant sur ruban magnétique pour s'abîmer ensuite dans son écoute au lieu de vivre pleinement le présent. Condamnées par l'indifférence des autres, elles s'écoutent donc parler et rire. Un tel repli sur soi menace leur amitié d'une atrophie de la communication en la réduisant au simple échange de cassettes.

Mais ces cassettes ne leur renvoient pas uniquement l'écho de leurs propres voix. Pour Chantal, elles sont aussi le réceptacle sacré où se répètent inlassablement des confidences précieuses échangées avec Fernand, le chauffeur d'autobus qu'elle retrouve chaque vendredi après-midi, le temps d'un trajet. Le rêve qu'elle poursuit avec cet homme pour lequel elle consent à ôter son baladeur, écran entre elle et les autres, est un rêve d'union parfaite. Ce rêve est inscrit dans la forme même de la sonate qui, dès le générique, se loge au cœur de l'univers diégétique. Cette sonate évoque la quête de l'harmonie puisqu'en elle, précise Gilbert Durand, s'épousent thème féminin et thème masculin (p. 401). La fonction du leitmotiv musical dans Sonatine est donc de rappeler que le rêve amoureux de Chantal se situe "hors discours", dans un espace sonore évoquant l'espace de la fusion corporelle avec la mère, celui de la non-séparation d'avant la crise du langage et l'interdiction œdipienne (Gorbman, p. 221). Aussi n'est-il pas surprenant que Fernand parle à peine et que Todor, le marin bulgare avec lequel Louisette passe une soirée à rêver, s'exprime dans une langue qu'elle ne comprend pas puisque, comme le raconte cette dernière en voix off, au moment où elle pleurait dans les bras du marin: "Il n'avait pas besoin de parler. Il avait juste besoin d'être là. ”

Grâce à cet étranger, il a suffi d'une nuit sur un bateau pour que Louisette, la mal aimée, se découvre précieuse, pour qu'elle se sente soignée, protégée. Ce qu'elle cherchait à vivre avec lui, elle le résume ainsi : "Je voulais voyager, qu'on s'aime sans parler en regardant le ciel. " Cette image du ciel en appelle une autre, celle du père. Comme l'expliquent Jean Chevalier et Alain Gheerbrant dans Le Dictionnaire des symboles: "Le père atteint une grandeur culturelle dans les mythes des origines; sa symbolique se confond alors avec celle du ciel et trahit le sentiment 
d'une absence, d'un manque, d'une perte, d'un vide que seul l'auteur des jours pourrait combler» (p. 741). Par ailleurs « [...] le père subsiste [...] toujours comme une image permanente de transcendance, qui ne peut être acceptée sans problème que par un amour réciproque d'âge adulte" (p. 741). Lélan qui pousse Chantal vers son chauffeur d'autobus et Louisette vers son marin bulgare correspond donc à leur besoin de transcendance incarnée par le père. Le père idéal pour les deux adolescentes serait le père procréateur, il redeviendrait cette "seconde mère" (Reed, p. 171) de la famille matrilinéaire. Comme Fernand, il offrirait du chocolat chaud à Chantal qu'il juge trop jeune pour boire du café; il l'inviterait à s'asseoir à la place la plus chaude dans l'autobus; comme le marin, ce père idéal bercerait Louisette lorsqu'elle pleurerait. Il serait, en un mot, plus guidé par l'anima que par l'animus. Avec un père comme celui-ci, l'être pourrait poursuivre son rêve de vie comme Chantal qui, après que Fernand lui ait souligné sa beauté, récite en voix off un poème où elle devient vague sortie de l'horizon, algue, ce symbole de "[...] vie sans limite et que rien ne peut anéantir" (Chevalier et Gheerbrant, p. 24). Le père idéal pourrait également repenser le monde, comme en cet instant où, sur un écran noir, s'élève la voix off de Chantal expliquant que le mot "grève" évoque chez elle le bord de mer. Lorsqu'à cette voix originelle, puisque jaillie du gouffre d'ombres, la voix de Fernand répond que «l'océan, c'est beau ", Chantal apparaît en gros plan, le baladeur collé aux oreilles. Elle est en train d'écouter une longue conversation qu'elle a eue avec son chauffeur préoccupé par la menace de grève des transporteurs du secteur public. En passant habilement de l'univers extra-diégétique (l'écran noir) à la diégèse (Chantal occupée à écouter sa cassette), la réalisatrice souligne comment son personnage masculin a pu, momentanément, se soustraire à l'emprise destructrice d'une société d'exploiteurs et d'exploités, en substituant à une image de répression l'image de l'océan, symbole "[...] d'indistinction primordiale [...] de dynamique de la vie" (Chevalier et Gheerbrant, p. 684). En écoutant la parole féminine portée par la rêverie marine, Fernand redécouvre une autre réalité, belle celle-là, chargée de promesses comme cet autre rêve que Chantal lui a confié dans 
lequel elle ressemblait à une plante en bourgeons, c'est-à-dire à jamais naissante puisque "[...] la plante, premier degré de la vie, symbolise surtout la naissance perpétuelle, le flux incessant de l'énergie vitale" (p. 764).

Ce flux, hélas, est brisé par un monde où les pères ne sont plus procréateurs, mais possesseurs, tout comme le premier patriarche. Ainsi, dans la seule scène où Louisette se retrouve avec ses parents, le rapport de dominant à dominé est inscrit de façon dramatique dans le discours filmique. Louisette, assise à table, quelque peu perdue dans le champ, mange. Ses parents la dominent nettement tandis qu'ils la contournent sans la voir, trop occupés qu'ils sont à se quereller au sujet de la grève des transports en commun. Sa position à droite dans l'image est pourtant celle où d'ordinaire tout personnage s'impose. Mais lorsque s'achève la séquence, le père debout, dos tourné à sa fille et à la caméra, a pris la place de Louisette désormais reléguée à gauche dans le champ. Cependant, comme dans la scène précédente où, en appelant vainement "Papa!», elle courait derrière le métro qui emportait son père et, comme le confirme ici l'usage métaphorique de la piste sonore, Louisette refuse de s'effacer devant une réalité économique qui la menace dans son être. Le bruit amplifié des céréales que Louisette, attablée, croque est tel qu'il recouvre presque complètement les propos de ses parents indifférents.

Cette résistance ne pourra toutefois tenir. Elle est aussi impossible qu'un amour pour un chauffeur d'autobus marié ou un marin inconnu. Le drame individuel de Louisette et de Chantal, privées de père "maternel», est clairement lié au drame collectif. Elles seront finalement vaincues par la grève des transports en commun qui, annoncée dès la première séquence, pèse sur la diégèse comme une fatalité. Elle est prévue pour novembre, identifié plus tard comme le mois des suicides. Cette grève est l'obsession du père de Louisette, conducteur de métro, qui en oublie jusqu'à l'existence de sa fille. Cette grève occupe aussi ces chauffeurs syndiqués qui souillent de leurs quolibets le rêve amoureux de Fernand et de Chantal alors qu'ils enjoignent leur camarade à se joindre à eux. Cette grève, enfin, prévient toute possibilité de sauvetage pour les adolescentes qui se sont empoi- 
sonnées dans le métro. Au moment où le conducteur va découvrir les corps affaissés de Chantal et de Louisette, il est arrêté net par le haut-parleur qui annonce que la grève a commencé: "Le métro est, dans un certain sens, responsable de la mort des filles"; plus encore: "Le métro, c'est le père. Et le père est la seule chose qui compte dans leur vie. Et le père est d'une certaine façon responsable de leur mort " (Lanctôt, citée dans Blain, p. 149). De même que le père est la force dominante, le métro est présenté dans le film comme une machine infernale dont le bruit envahit démesurément l'espace sonore. Il agresse de ses grincements métalliques, du crissement de ses pneus, du hurlement de sa sirène. Semblant tout droit venus de l'" Âge des métaux", cet âge guerrier, ces bruits s'opposent au murmure cristallin de l'eau d'un aquarium, semble-t-il, installé dans la chambre de Louisette, à la douce harmonie d'une sonatine véhiculée par des baladeurs, aux élans poériques de Chantal. Ce que dit clairement la piste sonore, c'est que, hors de leur univers intimiste, les deux adolescentes doivent se mesurer à un univers dur, industrialisé et fondé sur une inégalité profonde entre patrons et ouvriers. Cette inégalité en rappelle une autre, celle qui est apparue au moment où la possession communautaire a été remplacée par la propriété privée, période transitoire qui, chez certains peuples barbares, a été marquée par l'infanticide des filles (Reed, p. 214). La piste sonore du film nous ramène à cette tragédie historique où la femme est devenue la victime toute désignée d'un monde divisé entre exploiteurs et exploités. Alors que Louisette s'approche du bateau où elle va rencontrer son marin de rêve, elle longe un local d'où s'échappe la voix radiophonique d'un journaliste prévoyant une grève des transports publics après avoir annoncé le repêchage du corps d'une jeune fille dans le port de Montréal. Lorsque Louisette quittera le port, la séquence s'achèvera sur une vision d'apocalypse. Un autobus, ou serait-ce un bateau, brûle à l'arrière-plan. Le feu, ce principe de vie (Bachelard, 1949, p. 83), devient ici le feu guerrier, destructeur; quant à l'eau, elle est source de mort. Enfin, tandis que Louisette divise les pilules fatales, Chantal lit un poème de sa composition où il est question de la mer en feu. L'eau "[...] symbole profond, organique de la femme", selon 
Bachelard (1942, p. 113) est ici agressée, dominée par le feu, cette "mâle activité" (1949, p. 83). Dans un monde où l'animus triomphe de l'anima, la femme est condamnée à mort et cette mort n'a rien de glorieux.

Micheline Lanctôt n'utilise pas, en effet, ce que le critique Ian Lockerbie nomme le " [...] motif de l'apothéose [...] motif très typique de l'imaginaire québécois, aussi présent dans la littérature qu'au cinéma, qui consiste à présenter la mort d'un personnage comme une victoire" (p. 17). Les adolescentes de Sonatine ne meurent pas au cours d'une séquence au ralenti, emportées par les élans d'une composition classique, comme le Ti-Guy des Bons Débarras (Francis Mankiewicz, 1980). Contrairement à Réjean Ducharme, scénariste des Bons Débarras, Micheline Lanctôt n'inscrit pas son thème de la relève dans ce que Diane Pavlovic a nommé "[...] un scénario figuré, un scénario au figuré» (p. 85). Dans leur besoin de transcendance, les adolescentes de Sonatine ne se réfugient pas dans la littérature comme la jeune Manon des Bons Débarras. Elles se mesurent, elles, au quotidien précaire lorsque le rêve amoureux leur échappe.

Comme le précise Louise Carrière, les "[...] amours insolites pour un chauffeur d'autobus, un marin bulgare, permettent un moment d'oublier la réalité d'une société sclérosée par la peur et l'indifférence" (p. 113). Mais elle ajoute aussitôt: "Les adolescentes [...] ressemblent au moineau en cage apporté dans les bas-fonds par les mineurs. Elles aussi ont cessé de chanter et tentent désespérément d'alerter leurs semblables" (p. 113). Cette comparaison dramatique évoque un plan fixe du film, éloquent dans sa durée: le long plan où la caméra ne peut s'arracher au spectacle du violon abandonné par Louisette, au coeur du champ, sur le comptoir d'un guichet fermé de la station de métro. L'image de l'instrument qui ne chantera plus est le prélude à l'interminable séquence du suicide des adolescentes qui, là encore dans sa durée, semble vouloir signifier la détermination inébranlable de Louisette et Chantal. Assises dans le métro, avec leur pancarte implorant le monde indifférent pour qu'il les empêche de mourir, elles guettent le geste salvateur. Mais tous et toutes, jeunes et vieux entrent et sortent de la voiture sans réagir. Lorsque finalement une famille d'immigrants de 
race noire arrive et que le père s'approche pour lire l'appel au secours des jeunes filles, l'espoir renaît pour mourir aussitôt, le père se détourne et quitte la scène; le minoritaire ne reconnaît pas ses semblables parce qu'il ne les connaît plus. Alors, Louisette et Chantal choisissent la mort. Mais leur mort n'est pas renoncement. Elles lutteront jusqu'au bout contre le dernier sommeil. Chantal, la plus fragile, sombrera la première. Peu après, la tête de Louisette s'affaissera à son tour, alors que derrière la jeune fille vient de s'asseoir un musicien qui, après avoir lu la pancarte désespérée, la posera sans réagir.

En enfermant le suicide des adolescentes dans l'indifférence du minoritaire et de l'artiste, Micheline Lanctôt fait écho à la dénonciation de Denys Arcand qui, dans son documentaire $O n$ est au coton (1970), s'insurgeait contre "le confort et l'indifférence" (Jeancolas, p. 65) de ceux dont l'apathie entraînerait l'échec référendaire de l'année 1980. Dans cette mesure, l'agonie de Louisette et de Chantal ressemble au déclin progressif d'une culture menacée par la crise du monde capitaliste tout autant que par la fracture collective de ses origines. L'un des messages du film pourrait donc être celui que Ginette Major a identifié dans le cinéma québécois des années soixante-dix et qui dit peut-être "[...] jusqu'à éccurement ce que serait une société qui cesserait d'être une nation pour devenir une classe sociale perdue dans un univers anglo-américain" (p. 9). Mais la réalisatrice ne poursuit pas un rêve aussi étroitement nationaliste. Elle rêve d'unité, d'harmonie. Ce qu'elle semble dire à son peuple, c'est qu'il convient de dépasser le déterminisme historique pour accueillir le changement en renonçant à renouer avec un passé englué dans l'idéologie patriarcale qui mène le monde à sa perte. Elle invite les Québécois à ne plus vivre dans la nostalgie d'un Québec " [...] rural et harmonieux (et catholique) [...]" (Jeancolas, p. 73), à cesser de faire le jeu d'un système où ils sont devenus "[...] exploiteurs et exploités" (p. 73) pour rebâtir un monde en devenir constant, à l'image du rêve dynamique de Chantal et de Louisette qui trouvent leur point d'ancrage dans la mouvance d'un autobus et les départs d'un bateau. Ce rêve que sous-tend l'anima, cet "archétype de la vie " dont parle Jung (p. 62), est porté par deux jeunes femmes dont la voix, parce 
qu'elle est le plus souvent off, "[...] conserve une aura d'invulnérabilité et de puissance magique» (Chion, p. 33). Cette voix, partout baladée, elles l'ont enregistrée, marquant ainsi leur volonté exigeante et intransigeante d'inscrire la parole nouvelle, féminine, dans le devenir historique d'un peuple dont l'avenir menacé se confond à celui du monde occidental, puisqu'il entretient la survivance d'un système fondé sur des rapports de force où la femme aliénée et rompue ne peut plus être celle dont Aragon disait qu'elle est "l'avenir de l'homme".

\section{Mount Saint Vincent University}

\section{NOTES}

1 Les quelques indications placées entre guillemets apparaissent dans la deuxième version (datée de novembre 1981) du scénario de Sonatine écrit par Micheline Lanctôt et déposé aux archives de la Cinémathèque québécoise. Ces indications sont citées dans l'article de Gilles Blain, "Sonatine: un film à la recherche de son public?", dans Claude Chabot, Michel Larouche, Denise Pérusse et Pierre Véronneau (direction), Le Cinéma québécois des années 80 (Montréal: Cinémathèque québécoise, 1989), p. 134-151.

2 Ian Lockerbie rappelle que le sociologue Marcel Rioux a défini les trois grandes étapes de l'évolurion du Québec dans son livre La Question du Québec (Montréal: Parti Pris, 1980). Mais le critique précise également que quiconque se penche sur l'histoire québécoise peut distinguer "[...] l'étape historique de la conservation, l'étape du rattrapage de la modernité difficile et l'étape du dépassement, celle qui s'ouvre maintenant devant le Québec" ("Le cinéma québécois: une allégorie de la conscience collective", dans Claude Chabot, Michel Larouche, Denise Pérusse et Pierre Véronneau (direction), Le Cinéma québécois des années 80 (Montréal : Cinémathèque québécoise, 1989), p. 15.

\section{OUVRAGES CITÉS}

Bachelard, Gaston. L'Eau et les rêves. Paris: Librairie José Corti, 1942.

Bachelard, Gaston. La Psychanalyse du feu. Paris: Gallimard, 1949.

Blain, Gilles. "Sonatine: un film à la recherche d'un public?», dans Claude Chabot, Michel Larouche, Denise Pérusse et Pierre Véronneau (direction), Le Cinéma québécois des années 80. Montréal: La Cinémathèque québécoise (1989), p. 134-151.

Carrière, Louise. "Le long hiver des réalisatrices québécoises - 1970-1992 ", dans Sylvain Garel et André Pâquet (direction), Les Cinémas du Canada. Paris: Centre Georges Pompidou (1992), p. 103-115.

Chevalier, Jean et Alain Gheerbrant. Dictionnaire des symboles. Paris : Éditions Robert Laffont, 1969.

Chion, Michel. La Voix au cinéma. Paris : Éditions de l'Étoile, 1982.

De Candé, Roland. Dictionnaire de Musique. Paris: Microcosme / Seuil, 1982. 
Durand, Gilbert. Les Structures anthropologiques de l'imaginaire. Paris: Dunod, 1984. Gorbman, Claudia. Unheard Melodies: Narrative Film Music. Bloomington/Indianapolis: Indiana University Press, 1987.

Harcourt, Peter. "Naissance du direct 1960-1970", dans Sylvain Garel et André Pâquet (direction), Les Cinémas du Canada. Paris: Centre Georges Pompidou (1992), p. 51-63.

Jeancolas, Jean-Pierre. "La révolution par l'album - 1970-1980", dans Sylvain Garel et André Pâquet (direction), Les Cinémas du Canada. Paris: Centre Georges Pompidou (1992), p. 65-75.

Jung, Carl. G. Les Racines de la conscience. Paris: Editions Buchet-Chastel, 1971.

Lockerbie, Ian. "Le cinéma québécois: une allégorie de la conscience collective", dans Claude Chabot, Michel Larouche, Denise Pérusse et Pierre Véronneau (direction), Le Cinéma québécois des années 80. Montréal: La Cinémathèque québécoise (1989), p. 8-21.

Major, Ginetre. Le Cinéma québécois à la recherche d'un public. Bilan d'une décennie: 1970 -1980. Montréal : Les Presses de l'Université de Montréal, 1982.

Pavlovic, Diane. "Réjean Ducharme scénariste: les $\mathrm{p}(\mathrm{l})$ ans de l'illusion". Littérature québécoise et cinéma. Revue d'histoire littéraire du Québec et du Canada français, n" 11 (1986), p. 85-92.

Reed, Evelyn. Féminisme et Anthropologie. Paris : Denoël-Gonthier, 1979.

Vanasse, André. Le Pìre vaincu, la Méduse et les fils castrés. Montréal : XYZ éditeur, 1990.

Véronneau, Pierre. "Le Québec. De la fiction, dirent-ils - Des origines à 1960", dans Sylvain Garel et André Pâquet (direction), Les Cinémas du Canada. Paris: Centre Georges Pompidou (1992), p. 35-49. 